\title{
Ventilação não invasiva profilática, desfechos e seus impactos - uma revisão sistemática
}

\author{
Non-invasive prophylactic ventilation, outcomes and \\ their impacts - a systematic review
}

\section{Helder Brito Duarte', Kristine Menezes Barberino Mendes², Álec Paraíso Pereira³, Larissa Conceição Dias ${ }^{4}$, Camilla de Souza Menezes ${ }^{5}$}

${ }^{1}$ Autor para correspondência. Universidade Salvador, Hospital da Cidade. Salvador, Bahia, Brasil. ORCID: 0000-0002-4656-750X. helderphysio@gmail.com

${ }^{2}$ Hospital da Cidade. Salvador, Bahia, Brasil. ORCID: 0000-0001-5933-5305. kbarberino@gmail.com ${ }^{3}$ Escola Estadual de Saúde Pública, Hospital Geral Roberto Santos. Salvador, Bahia, Brasil. ORCID: 0000-0001-8209-8520. alecparaiso@gmail.com ${ }^{4}$ Escola Estadual de Saúde Pública, Hospital Geral Roberto Santos. Salvador, Bahia, Brasil. ORCID: 0000-0002-7648-4879. cdias.larissa@gmail.com ${ }^{5}$ Fundação Estatal Saúde da Família, Fundação Oswaldo Cruz. Salvador, Bahia, Brasil. ORCID: 0000-0002-4617-1396. millaa_menezes@hotmail.com

RESUMO | INTRODUÇão: Ventilação Não Invasiva Profilática (VNIP) é uma modalidade de VNI aplicada a pacientes que foram eleitos para extubação, porém possui alto risco de falha. OBJETIVO: descrever e analisar populações favorecidas pela VNIP, impactos e desfechos. MÉTODO: Revisão sistemática, buscaram-se ensaios nas bases de dados PubMed, EMBASE e Cochrane Library com os seguintes critérios de inclusão: publicação nos últimos 20 anos, escrito em inglês, espanhol ou português; idade $\geq 18$ anos; ventilação mecânica $\geq 48$ horas; comparação da VNIP com terapia padrão. Critérios de exclusão: VNIP < 4 horas; e VNIP intermitente. RESULTADOS: Resultaram-se oito estudos. Os desfechos foram: reintubação, Insuficiência Respiratória Pós Extubação (IRPE), mortalidade e permanência na UTI. Características relevantes extraídas: uso de VNIP de resgate, níveis de $\mathrm{PaCO} 2$, pressão inspiratória máxima e índice de respiração rápida superficial. Os estudos relatam que a aplicação de VNIP em população heterogênea reduz o risco de desenvolver IRPE, porém parece não haver consistência referente à reintubação, mortalidade na UTI, permanência na UTI e hospitalar. Estes marcadores foram apresentados através de resultados controversos entre os estudos revisados. Em pneumopatas especificamente, VNIP parece não ter impacto direto em reintubação e permanência na UTI. CONCLUSÃO: Os estudos divergem sobre uso da VNIP em prevenir principais desfechos, mesmo em pneumopatas, porém, seu uso em evitar IRPE é positivo. Sendo assim, necessitam-se estudos em populações com melhor predisposição a sucesso na extubação para comprovar a eficácia da VNIP.

PALAVRAS-CHAVE: Ventilação não invasiva. Extubação. Insuficiência respiratória.
ABSTRACT | INTRODUCTION: Noninvasive Ventilation Prophylactic (NIVP) is a modality of NIV applied to patients who have planned extubation but has a high risk of failure. OBJECTIVE: The objective of this study was to describe and analyze populations favored by NIVP, impacts and outcomes. MATERIAL AND METHODS: For this, essays were searched in the Pubmed, EMBASE and Cochrane Library databases with the following inclusion criteria: publication in the last 20 years, written in English, Spanish or Portuguese; age $\geq 18$ years; mechanical ventilation $\geq 48$ hours; and compare NIVP with standard therapy. Exclusion criteria: NIVP $<4$ hours and intermittent NIVP. RESULTS: Eight studies were resulted. The outcomes were: reintubation, Respiratory Failure Post Extubation (RFPE), mortality and ICU permanence. Relevant features extracted: use of rescue NIV, PaCO2 levels, maximal inspiratory pressure and rapid superficial respiration index. Studies report that the application of NIVP in a heterogeneous population reduces the risk of developing RFPE, but there seems to be no consistency regarding reintubation, ICU mortality, permanence in the ICU and hospital. These markers were presented through controversial results among the reviewed studies. In lung disease specifically, NIPV seems to have no direct impact on reintubation and ICU stay. CONCLUSIONS: It is possible conclude that the present studies differ on the use of NIPV to prevent major outcomes such as reintubation and mortality, even in lung disease, but its use in preventing RFPE is positive. Therefore, studies in populations with better predisposition to successful extubation are necessary to prove the efficacy of NIPV.

KEYWORDS: Noninvasive ventilation. Extubation. Respiratory failure. 


\section{Introdução}

A extubação do paciente crítico é um fator importante em sua trajetória clínica e, além de mais precoce possível, deve ser realizada de forma segura e eficaz. Contudo, a falha de extubação pode ocorrer entre 6 e $23 \%$ das extubações planejadas ${ }^{1,2}$. Este fator pode ser originário da Insuficiência Respiratória PósExtubatória (IRPE) que, na maioria das vezes é resultante de obstrução em via aérea, tosse inadequada, secreções copiosas, encefalopatia e disfunção cardíaca. Ainda assim, outros fatores como idade e pneumopatia podem aumentar as chances de falha ${ }^{2-4}$.

A reintubação resulta em piora do prognóstico do paciente devido à risco de pneumonia nosocomial e mortalidade aumentada, sendo assim, para evitar este evento, a ventilação não invasiva (VNI) é sugerida a depender do quadro do paciente ${ }^{1,2}$.

A VNI pós-extubatória pode ser associada a três grupos: facilitadora, resgate e profilática. VNI Facilitadora é aplicada em pacientes que falharam no Teste de Respiração Espontânea (TRE) porém, com a causa de intubação resolvida, são extubados e iniciado o suporte não invasivo. Esta modalidade possui evidências sólidas com ensaios clínicos bem consolidados e resultados favoráveis à sua aplicação com melhora da sobrevida e redução de tempo de Ventilação Mecânica (VM) em populações com Doença Pulmonar Obstrutiva Crônica (DPOC) ${ }^{5,6}$.

A VNI de resgate é utilizada quando o paciente é extubado e já possui o diagnóstico de IRPE instalado. Seu uso não possui evidências de impacto em tempo de ventilação mecânica ou mortalidade e pode piorar o prognóstico do paciente se postergada a reintubação. Ao contrário das citadas anteriormente, a VNI Profilática (VNIP) é utilizada principalmente nos grupos de pacientes de alto risco para falha da extubação mesmo possuindo resultados favoráveis no TRE ${ }^{2,3,6}$.

A partir desta observação, a presente revisão sistemática tem como objetivo descrever quais populações em estado crítico após extubação eletiva que se beneficiam com VNIP bem como a análise de marcadores e o impacto da VNIP nos seus desfechos.

\section{Método}

Este estudo consiste em uma revisão sistemática da literatura seguindo os preceitos do guia Transparent Reporting of System Reviews and Meta-analyses PRISMA. Para isso, foram realizadas buscas de ensaios clínicos randomizados nas bases de dados Pubmed, EMBASE e Cochrane Library respeitando os seguintes critérios: período publicado entre Janeiro de 1990 a Agosto de 2019; estar escrito na íntegra em inglês, espanhol ou português; idade $\geq 18$ anos, formato de ensaio clínico, comparação VNIP com terapia padrão (oxigenoterapia e terapias medicamentosas). Foram excluídos os estudos que tinham aplicação de VNIP menor que 4 horas e VNIP intermitente.

Para retornar resultados de forma filtrada, os termos obtidos através do Mesh terms foram combinados com as palavras "OR" (sinônimo), "AND" (soma) e "NOT" (negação) em uma sintaxe. Os termos determinados foram: "Noninvasive ventilation", "Continuous Positive Airway Pressure", "extubation", excluindo os termos "preterm", "newborn", "infants" e "children".

A busca sistemática foi feita por três autores, foi dividida em 5 fases (conforme consta na Figura 01): a primeira fase foi a busca nas bases de dados citadas anteriormente com a sintaxe formulada com finalidade de reunir referências ligadas ao tema e, concomitantemente, foi feita uma busca na literatura cinza para agregar referências não publicadas. Esta busca resultou em 427 referências nas bases convencionais e nenhuma na literatura cinza. A segunda fase se deu pela eliminação das referências duplicadas resultando em 415 referências. Na fase seguinte, a terceira, foi feita a filtragem dos artigos após a leitura do título e resumo na íntegra. Caso houvesse conflitos na seleção das referências, houve discussão entre os três autores para definir a inclusão ou não. Nesta fase foram excluídas 398 referências por não se adequar aos critérios de inclusão. Já a quarta fase consistiu na aplicação dos critérios de exclusão para uma melhor uniformidade dos artigos, nesta excluiu-se 10. A quinta fase foi a extração e síntese dos dados dos 7 artigos. Esta etapa foi realizada por dois autores. 


\section{Resultados}

Os 7 artigos situavam-se entre os períodos de 2005 e 2013. Suas amostras continham de 38 a 406 pacientes. A maioria deles avaliaram populações heterogêneas, no entanto, dois estudaram a utilização da técnica em pneumopatas. O tempo de aplicação da VNI variou entre 8 horas por dia a 48 horas com pausas apenas para higiene pessoal e alimentação. A única semelhança nos estudos foi a terapia padrão que estava associada a oxigenoterapia, porém com oferta diferente.

Figura 1. Fluxograma da busca sistemática de estudo em população crítica em uso VNIP pós-extubação eletiva 2019
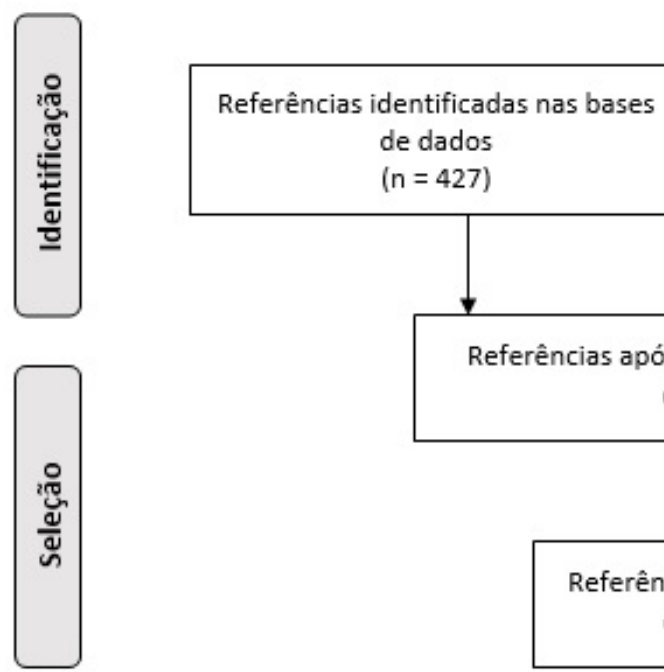

Referências após remoção de duplicatas $(n=415)$
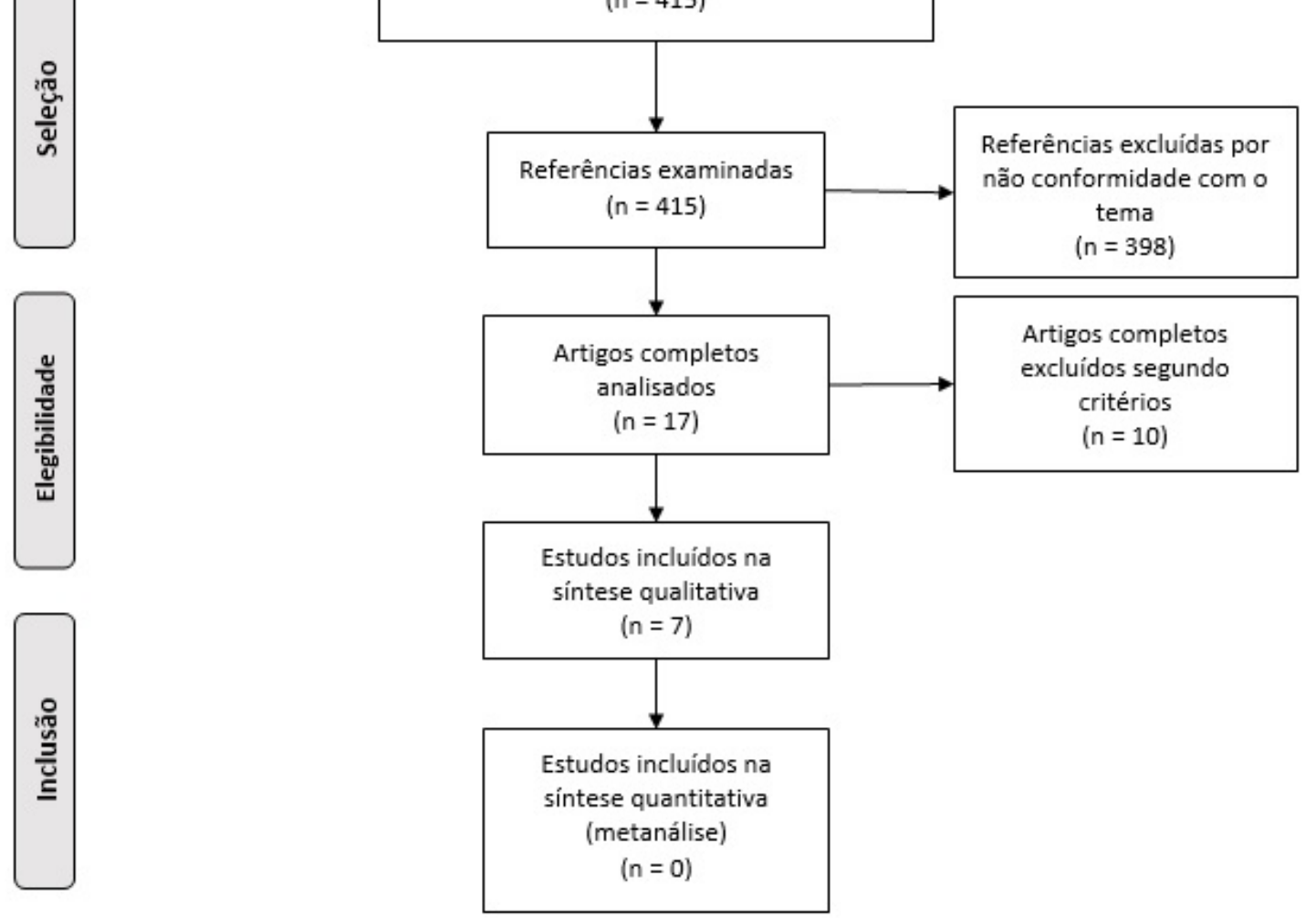

A avaliação da qualidade metodológica consistiu na utilização da escala da base de dados Physiotherapy Evidence Database (PEDro) constatando três ensaios nível 5/10, três 6/10 e um 7/10. Mesmo havendo estudos com qualidade moderada isto não implicou negativamente na análise de dados. Para a análise primária, foram extraídos e expostos na Tabela 1 os dados sobre: reintubação, insuficiência respiratória pós extubação, mortalidade e permanência na UTI, (Tabela 1). 
Tabela 1. Descrição dos ensaios clínicos randomizados em população crítica em uso VNIP pós-extubação eletiva 2019

\begin{tabular}{|c|c|c|c|c|c|}
\hline Autor & população & $\begin{array}{l}\text { Amostra } \\
\text { (VNI:Controle) }\end{array}$ & Duração VNI & Qual. & Desfecho \\
\hline $\begin{array}{l}\text { Nava S, et } \\
\text { al., } 2005^{3}\end{array}$ & Heterogênea & $97(48: 49)$ & $8 \mathrm{~h} / \mathrm{dia}$ por $48 \mathrm{~h}$ & $5 / 10$ & $\begin{array}{l}\text { VNI diminuiu reintubação e na } \\
\text { mortalidade }\end{array}$ \\
\hline $\begin{array}{l}\text { Ferrer } \mathrm{M} \text {, et } \\
\text { al., } 2006^{4}\end{array}$ & $\begin{array}{l}\text { Idoso, } \\
\text { cardiopata }\end{array}$ & 162(79:83) & $19 \mathrm{~h}$ & $7 / 10$ & $\begin{array}{l}\text { VNI diminuiu IRpA em } 24 \text { h pós } \\
\text { extubação. }\end{array}$ \\
\hline $\begin{array}{l}\text { Ferrer M, et } \\
\text { al., } 2009^{2}\end{array}$ & $\begin{array}{l}\text { Pneumopata } \\
\text { com PCO2 } \\
>45 \text { durante } \\
\text { TRE }\end{array}$ & $106(54: 52)$ & $18 \mathrm{~h}$ & $6 / 10$ & $\begin{array}{l}\text { VNI diminuiu IRpA em } 24 \text { h pós } \\
\text { extubação, redução de mortalidade } \\
\text { em } 90 \text { dias }\end{array}$ \\
\hline $\begin{array}{l}\text { Khilnani G } \\
\text { et al., } 2011^{7}\end{array}$ & $\begin{array}{l}\text { DPOC } \\
\text { hipercápnico }\end{array}$ & $40(20: 20)$ & $34,7 \mathrm{~h}$ & $5 / 10$ & $\begin{array}{l}\text { Sem alterações significativas em } \\
\text { reintubação e alta hospitalar }\end{array}$ \\
\hline $\begin{array}{l}\text { Su CL, et al., } \\
2012^{8}\end{array}$ & Heterogênea & $406(204: 202)$ & $12 \mathrm{~h}$ & $6 / 10$ & $\begin{array}{l}\text { Sem alterações significativas em } \\
\text { Reintubação e mortalidade }\end{array}$ \\
\hline $\begin{array}{l}\text { Mohamed } \\
\text { K. et al., } \\
2013^{9}\end{array}$ & Heterogênea & $120(60: 60)$ & $12 \mathrm{~h}$ & $6 / 10$ & $\begin{array}{l}\text { VNI diminuiu reintubação, inclusive } \\
\text { em DPOC, reduziu tempo de } \\
\text { permanência hospitalar e índice de } \\
\text { PAV }\end{array}$ \\
\hline $\begin{array}{l}\text { Ornico S, et } \\
\text { al., } 2013^{10}\end{array}$ & Heterogênea & $38(20: 18)$ & $24 \mathrm{~h}$ & $5 / 10$ & $\begin{array}{l}\text { VNI diminuiu reintubação, } \\
\text { mortalidade e tempo de } \\
\text { permanência na UTI }\end{array}$ \\
\hline
\end{tabular}

Legenda: VNI: Ventilação Não Invasiva; Qual.: Qualidade; IRpA: Insuficiência Respiratória Aguda; DPOC: Doença Pulmonar Obstrutiva Crônica; PAV: Pneumonia Associada à Ventilação Mecânica

Os dados secundários como uso de VNI de resgate, níveis de PaCO2, pressão inspiratória máxima (PiMAX) e índice de respiração rápida superficial (IRRS) foram também captados e distribuídos na tabela 2, além de mostrar quais foram as pressões utilizadas e os principais resultados dos estudos pesquisados. Dentre estes valores pode-se observar a variação entre as pressões inspiratória empregadas, entre 10 e 14 em média, sendo titulada pelos estudos a partir da necessidade do paciente em obter faixas seguras de $\mathrm{pH}$, trabalho ventilatório e $\mathrm{PaCO} 2$ dos pacientes. Variáveis de desfechos também foram coletadas em quantidade e porcentagem de pacientes. Estas variáveis foram: reintubações, Insuficiência respiratória pós extubação e mortalidade na UTI. Já os dados de tempo de permanência na UTI e hospitalar foram computados em média e desvio padrão.

Segundo os dados coletados, o índice de reintubação reduzido obteve resultados favoráveis em 3 estudos ${ }^{3,9,10}$ de 7, seguido por diminuição da mortalidade (3 estudos ${ }^{4,9,10} \mathrm{em} \mathrm{6}$ ) e permanência na UTI (1 estudo ${ }^{9}$ de 6). 0 índice de IRPE foi pouco exposto nos ensaios, porém, dos 3 estudos, em $2^{2,4}$ este marcador resultou em sucesso da VNI em comparação com o grupo controle (Tabela 2). 
Tabela 2. Descrição principais desfechos no estudo em população crítica em uso VNIP pós-extubação eletiva 2019

\begin{tabular}{|c|c|c|c|c|c|}
\hline Autor & $\begin{array}{l}\text { Parâmetros } \\
\text { Média(DP) }\end{array}$ & RelOT* & IRPE* & Óbito UTI* & Permanência UTI* \\
\hline Nava S, et & Pi: $13,1(4,5)$ & $4(8): 12(24)$ & NA & $3(6): 9(18)$ & $8,9(5,7): 11,6(14,9)$ \\
\hline al., $2005^{3}$ & Pe:5,2(1,6) & $p=0,027$ & & $p=0,064$ & \\
\hline Ferrer $\mathrm{M}$, et & Pi 14(3) & $9(11): 18(22)$ & $13(16): 27(33)$ & $2(3): 12(14)$ & 11(8):13(11) \\
\hline al., $2006^{4}$ & $\operatorname{Pe} 5(1)$ & $p=0,12$ & $p=0,029$ & $p=0,015$ & $p=0,14$ \\
\hline Ferrer $\mathrm{M}$, et & Pi: 12 a 20 & $6(11): 10(19)$ & $8(15): 25(48)$ & $3(6): 4(8)$ & 11(13):10(9) \\
\hline al., $2009^{2}$ & Pe: 5 a 6 & $p=0,3741$ & $p<0,0001$ & $p=0,7132$ & $p=0,5041$ \\
\hline Khilnani G & Pi: $12,1(1,3)$ & $3(15): 5(20)$ & NA & NA & $2,0(2,1): 1,5(0,8)$ \\
\hline et al., $2011^{7}$ & Pe: $6,5(1)$ & $p=0,44$ & & & $p=0,34$ \\
\hline Su CL, et & Pi: 10 a 12 & $21(10): 16(7,7)$ & $27(13,2): 30$ & $2(0,9): 3(1,4)$ & NA \\
\hline al., $2012^{8}$ & Pe: 5 & $p=0,37$ & $(14,9) p=0,62$ & $p=0,64$ & \\
\hline Mohamed & Pi: 10(2) & $9(15): 15(25)$ & NA & $4(6,6): 10(16,6)$ & $8,3(3,1): 11,6(2,6)$ \\
\hline $\begin{array}{l}\text { K. et al., } \\
2013^{9}\end{array}$ & Pe: 5 & $P=0,049$ & & $p=0,047$ & $p=0,04$ \\
\hline Ornico S, et & $\mathrm{Pi}: 12$ & 1(5):7(39) & NA & $0(0): 4(22,2)$ & $16,8(11,6): 18,4(12,2)$ \\
\hline al., $2013^{10}$ & Pe: 8 & $p=0,016$ & & $p=0,041$ & $p=0,681$ \\
\hline
\end{tabular}

Legenda:* VNI vs Controle(\%); DP: Desvio Padrão; NA: Não Avaliado; IRPE: Insuficiência Respiratória Pós Extubação; VNI: Ventilação Não invasiva; Pi: Pressão Inspiratória; Pe: Pressão Expiratória

Outros desfechos secundários das pesquisas foram transcritos na tabela 3. Esses desfechos são relacionados ao uso de $\mathrm{VNI}$ de resgate, $\mathrm{PaCO} 2$ mensurada durante o TRE ou na pós extubação, Permanência hospitalar, PiMAX e IRRS. O uso da VNI de resgate foi uma prática realizada por 3 autores ${ }^{2,4,8}$, idealizada no intuito de evitar reintubação dos pacientes que cursaram com insuficiência respiratória pós extubação. A variável $\mathrm{PaCO} 2$ alternou entre valores de normalidade $(37 \mathrm{mmHg}$ ) e valores elevados $(82,3$ $\mathrm{mmHG}$ ), não havendo diferença estatística entre os grupos. Permanência hospitalar também não obteve sucesso em diferença estatística, variando resultados entre 16,1 e 30 dias no grupo VNI e 18,2 e 29 dias no grupo controle.
Poucos estudos relataram os valores de PiMAX e IRRS, porém, todos afirmaram não haver diferença significativa entre os grupos VNI e controle. Apenas um estudo ${ }^{8}$ diferenciou esses valores destas variáveis entre pacientes que falharam na extubação (PiMAX $-27,7 \pm 0,9$ e $-32,4 \pm 1,5$ e IRRS $73,3 \pm 4,6$ e $80,1 \pm 4,3$, VNI e IRRS respectivamente) e os que tiveram sucesso (PiMAX $-35,2 \pm 0,9$ e $-35,2 \pm 0,9$ e IRRS $58,6 \pm 1,8$ e $58,0 \pm 1,7, \mathrm{VNI}$ e controle respectivamente). Na variável PiMAX, o grupo VNI apresentou diferença estatística entre sucesso e falha $(p=0,01)$, não havendo resultado semelhante no grupo controle $(p=0,23)$. Em IRRS, ambos grupos apresentaram diferença estatística ( $p=0,02$ e $p<0,001, \mathrm{VNI}$ e controle respectivamente). 
Tabela 3. Descrição de dados secundários em população crítica em uso VNIP pós-extubação eletiva 2019

\begin{tabular}{|c|c|c|c|c|c|}
\hline Autor & VNIR & PaCO2 Média(DP)* & $\begin{array}{l}\text { Perm. Hosp. Média } \\
\text { (DP)* }\end{array}$ & PiMAX* & IRRS (f/Vt)* \\
\hline $\begin{array}{l}\text { Nava S, et al., } \\
2005^{3}\end{array}$ & Não & (Pós) 41,7(4,2):39,4(5,6) & $\begin{array}{l}23,3(16,4): 25,5 \\
(21,4) p=?\end{array}$ & NA & NA \\
\hline $\begin{array}{l}\text { Ferrer M, et al., } \\
2006^{4}\end{array}$ & Sim & (TRE) 38(5):37(5) & $\begin{array}{l}30(23): 29(18) \\
p=0,65\end{array}$ & NA & NA \\
\hline $\begin{array}{l}\text { Ferrer M, et al., } \\
2009^{2}\end{array}$ & Sim & (TRE) 55(6):53(5) & $\begin{array}{l}29(27): 25(17) \\
p=0,2988\end{array}$ & NA & $66(26): 65(23)$ \\
\hline $\begin{array}{l}\text { Khilnani G et al., } \\
2011^{7}\end{array}$ & Não & $\begin{array}{l}\text { (Pós)82,3(11,2): } \\
80,0(13,2)\end{array}$ & $\begin{array}{l}16,1(6,2): 18,2 \\
(7,9) \text { p } 0,34\end{array}$ & NA & NA \\
\hline $\begin{array}{l}\text { Su CL, et al., } \\
2012^{8}\end{array}$ & Sim & NA & NA & $\begin{array}{l}35,2(0,9): 27,7(0,9) \\
(p=0,01) \text { vs } \\
35,2(0,9): 32,4(1,5) \\
(p=0,23) \mathbb{P}\end{array}$ & $\begin{array}{l}58,6(1,8): 58,0 \\
(1,7)(p=0,02) \text { vs } \\
73,3(4,6): \\
80,1(4,3) \\
(p<0,001) \mathbb{P}\end{array}$ \\
\hline $\begin{array}{l}\text { Mohamed K. et } \\
\text { al., } 2013^{9}\end{array}$ & Não & (Pós) 39.9(6,3):42,8 (7,7) & NA & $33(11,6): 34(13,7)$ & $\begin{array}{l}93,9(31,8): \\
95,9 \\
(34,1)\end{array}$ \\
\hline $\begin{array}{l}\text { Ornico S, et al., } \\
2013^{10}\end{array}$ & Não & NA & NA & NA & NA \\
\hline
\end{tabular}

Legenda: DP: Desvio Padrão; VNI: Ventilação Não Invasiva; * Sucesso versus Falha; l: VNI versus Controle; Pós: Pós Extubação; TRE: Treino de Respiração Espontânea; PiMAX: Pressão Inspiratória Máxima; IRRS: Índice de Respiração Rápida Superficial

Alguns estudos $2,4,7,9$ remeteram sua atenção total ou parcial para população de pneumopatas fazendo-se necessário a inclusão desses dados na tabela 4 a qual discorre sobre reintubação, Insuficiência Respiratória pós extubação, mortalidade na UTI, permanência na UTI e mortalidade hospitalar. Estes estudos mostram não haver diferença estatística em reintubação e permanência na UTI entre os grupos VNI e controle, porém, os resultados referentes a Insuficiência Respiratória pós extubação, mortalidade em UTI e mortalidade hospitalar mostraram-se controversos.

Tabela 4. Resultados em pneumopatas em população crítica em uso VNIP pós-extubação eletiva 2019

\begin{tabular}{|c|c|c|c|c|c|}
\hline Autor & RelOT* & IRPE* & Óbito UTI* & Perm. UTI* & Óbito hospitalar (dias)* \\
\hline $\begin{array}{l}\text { Ferrer M, et al., } \\
2006^{4}\end{array}$ & $\begin{array}{l}3(11): 6(27) \\
p=0,27\end{array}$ & $\begin{array}{l}4(15): 9(41) \\
p=0,083\end{array}$ & $\begin{array}{l}0(0): 4(18) \\
p=0,035\end{array}$ & NA & $1(4): 9(41) p=0,003$ \\
\hline $\begin{array}{l}\text { Ferrer M, et al., } \\
2009^{2}\end{array}$ & $\begin{array}{l}6(11): 10(19) p \\
=0,3741\end{array}$ & $\begin{array}{l}8(15): 25(48) \\
p<0,0001\end{array}$ & $\begin{array}{l}3(6): 4(8) \\
p=0,7132\end{array}$ & $\begin{array}{l}11(13): 10(9) \\
p=0,50\end{array}$ & $6(11): 11(22) p=0,25$ \\
\hline $\begin{array}{l}\text { Khilnani G et } \\
\text { al., } 2011^{7}\end{array}$ & $\begin{array}{l}3(15): 5(20) \\
p=0,44\end{array}$ & NA & NA & $\begin{array}{l}2,0(2,1): 1,5(0,8) \\
p=0,34\end{array}$ & NA \\
\hline $\begin{array}{l}\text { Mohamed K. et } \\
\text { al., } 2013^{9}\end{array}$ & $\begin{array}{l}1(5,25): 5(31,2) \\
p=0,019\end{array}$ & NA & NA & NA & NA \\
\hline
\end{tabular}




\section{Discussão}

Em populações heterogêneas, a análise dos estudos sugere que a VNIP diminui o risco de IRPE, porém, não tem consistência em prevenir a reintubação, diminuir a taxa de mortalidade na UTI e reduzir tempo de permanência na UTI e hospitalar, visto que estes marcadores são apresentados através de resultados controversos nos estudos revisados.

$\mathrm{Na}$ população de pneumopatas especificamente, a VNIP não sugeriu impacto em prevenir reintubação e diminuir tempo de permanência na UTI se comparado com o grupo controle. Nas variáveis IRPE, mortalidade na UTI e hospitalar apresentou resultados inconsistentes. A indicação da VNIP variou de acordo com os estudos, porém, todos eles são unânimes em eleger pacientes com VM por mais de 48 horas, tendo como causa da intubação a Insuficiência respiratória aguda do tipo ${ }^{2,4,9-10}$ ou tipo $\mathrm{II}^{7}$ somado ao sucesso no TRE. Alguns autores determinaram que pneumopatas ${ }^{2,7}$ ou populações com alto risco de reintubação ${ }^{3,4}$ se beneficiariam com a VNIP trazendo desfechos positivos.

Um estudo3 configurou a população de alto risco como: VM por mais de 48 horas, TRE com sucesso, um ou mais dos critérios de alto risco de reintubação: Mais que uma falha consecutiva no teste de respiração espontânea; Insuficiência cardíaca crônica, $\mathrm{PaCO} 2>45 \mathrm{mmHg}$ após extubação, mais que uma comorbidade (excluindo Insuficiência Cardíaca Crônica); tosse ineficaz; estridor de vias aéreas superiores não requerendo intubação imediata. Já outro ensaio clínico4, reuniu critérios como: idade maior que 65 anos, Insuficiência cardíaca como causa da intubação ou gravidade aumentada traduzida através do escore Acute Physiology and Chronic Health Evaluation (APACHE)-II maior que 12.

O principal desfecho encontrado em todos estudos foi a reintubação. Apenas três estudos ${ }^{3,9,10}$ encontraram resultados estatisticamente significativos para prevenção de reintubação. Alguns autores ${ }^{2,4}$ justificam como as principais causas de reintubação a insuficiência respiratória (principalmente por aumento da PaCO2), excesso de secreção em via aérea e insuficiência cardíaca.
Alguns desses estudos ${ }^{2,4,8}$, realizaram VNI de resgate em ambos os grupos que não necessitaram de intubação imediata. Os pacientes alocados no grupo VNIP receberam este suporte após finalizarem o protocolo de intervenção e desenvolveram, em 72 horas, insuficiência respiratória pós extubação. Já no grupo controle os pacientes que cursaram com esta afecção em até 72 horas, foi aplicado a modalidade VNI de resgate.

A reintubação em muitos casos precede a IRPE. Esta variável foi estudada por três autores ${ }^{2,4,8}$ e observaram resultados positivos para o uso da VNIP. Estes autores acreditam que, com a aplicação da modalidade VNI de Resgate naqueles que não precisavam ser reintubados imediatamente, houve uma reversão em parte dos casos de IRPE o que pode explicar a não significância estatística da variável reintubação nestes estudos. Dois ensaios clínicos 2,4 quantificaram estes valores de forma específica e constataram a eficácia em $100 \%$ e $35 \%$ versus $47 \%$ e $35 \%$ de reversão da insuficiência respiratória nos grupos $\mathrm{VNI}$ e controle respectivamente, reduzindo assim o índice de reintubação.

Outros fatores como PaCO2, PiMAX e IRRS podem ter contribuído para as falhas na extubação. Khilnani, et al. 7 recrutou pacientes com PaCO2 média de 82,3 e $80,0 \mathrm{mmHg}$ (grupos VNI e controle, respectivamente) com pH de 7,22, semelhante nos dois grupos. Este não obteve resultados positivos no uso da VNIP, acredita-se que valores de $\mathrm{PaCO} 2$ acima de 60 somado a $\mathrm{pH}$ entre 7,20 e 7,25 podem elevar as possibilidades de reintubação ${ }^{12}$.

A variável PiMAX foi estratificada nas populações que obtiveram sucesso e falha na extubação. Su CL et al. ${ }^{8}$ afirmou haver alteração significativa desses valores no grupo $\mathrm{VNI}(-35,2 \pm 0,9$ e- $27,7 \pm 0,9 p=0,01$ sucesso e falha, respectivamente) porém, no grupo controle não foi observada alterações significativas nesta variável $(-35,2 \pm 0,9$ e $-32,4 \pm 1,5 p=0,23$ sucesso e falha, respectivamente). Acredita-se que, segundo um estudo anterior ${ }^{13}$, a PiMAX menor que $30 \mathrm{cmH} 2 \mathrm{O}$ é um preditor importante para falha de extubação do paciente, podendo ser este um dos fatores que levaram uma parte do grupo VNI à reintubação. 
O IRRS também foi estratificado pelo autor citado anteriormente8, o que foi constatado diferença estatística em termos de sucesso e falha nos resultados dos grupos VNI $(56,6 \pm 1,8$ e $73,3 \pm 4,6 p=0,02)$ e controle $(58,0 \pm 1,7$ e $80,1 \pm 4,3 p<0,001)$. Esta diferença acentuada entre os dois grupos pode estar relacionada com a proximidade dos valores a 100 respirações/L, ou seja, quanto mais próximo os valores de 100 respirações/L, mais suscetível a falha de extubação desses pacientes ${ }^{14}$. Porém, Mohamed, et al. ${ }^{9}$ mesmo com IRRS em seu estudo com resultados de 93,9 $9 \pm 31,8$ e $95,9 \pm 34,1$ nos grupos VNI e controle, respectivamente, favoreceu resultados positivos no uso da VNIP impactando em reintubação, mortalidade na UTI e permanência na UTI. Este resultado de IRRS pode estar relacionado com o auxílio ventilatório que a VNIP, por ter valores próximos a 100 respirações/L, proporcionou aos pacientes deste grupo. Já no estudo realizado por Ferrer, et al. ${ }^{2}$, seus valores de IRRS foram de $66 \pm 26$ e $65 \pm 23$ nos grupos VNI e controle, respectivamente, porém os resultados na variável reintubação foram semelhantes, podendo ser explicado por outra causa durante $72 \mathrm{~h}$ após a extubação.

Referente à redução da mortalidade na UTI, a grande maioria dos estudos ${ }^{2,3,8}$ apontaram que não houve diferença estatística entre os grupos, três autores conseguiram resultados positivos ${ }^{4,9,10}$ nesta variável, sendo assim estes achados podem ser explicados através dos índices de reintubação reduzidos no grupo $\mathrm{VNI}$ em dois estudos ${ }^{9,10}$. Ao contrário, os demais autores podem não ter achado relevância nos dados devido ao aumento da gravidade dos pacientes que são reintubados.

Em pneumopatas, a grande maioria dos autores ${ }^{2,4,7}$ não encontraram diferença estatística que sustentam a intervenção. Ao contrário desses achados, Burns e colaboradores $^{14}$ em sua revisão sistemática, constatou que pacientes com DPOC se beneficiam da VNI facilitadora (que auxiliam no processo de desmame da prótese ventilatória) proporcionando redução em mortalidade e pneumonia nesses pacientes. Nesta revisão sistemática, que utiliza VNIP, esses achados foram inconsistentes.

Esta revisão sistemática possui algumas limitações: baixo número de artigos reunidos. Mesmo com o cuidado na formulação de uma sintaxe apropriada e busca na literatura cinza, esta revisão contou apenas com sete ensaios clínicos; poucas variáveis relatadas em todos estudos; a comparação dos desfechos, apenas índice de reintubação foi avaliado em todos estudos; protocolos diversos de aplicação da VNIP; não há descrito na literatura sobre uma padronização na aplicação da técnica (início, duração e uso ou não de VNI de resgate), isto pode ter impactado nos desfechos analisados.

\section{Conclusão}

Esta revisão sistemática sugere que VNIP reduz insuficiência respiratória pós-extubação, porém não apresenta impacto na prevenção de reintubação em populações heterogênea e pneumopatas. Outros desfechos como mortalidade, permanência na UTI e tempo de permanência hospitalar não apresentaram dados consistentes. Portanto, para solidificar esta prática nas rotinas das UTIs, é importante a realização de novos estudos a fim de determinar populações específicas que podem se beneficiar diretamente com a VNIP.

\section{Agradecimentos}

Este estudo foi apresentado no VII Congresso Luso-Brasileiro de medicina intensiva e I Simpósio de Cuidados ao Paciente crítico do Hospital da Cidade (Premiado em $2^{\circ}$ lugar).

\section{Contribuições dos autores}

Duarte HB redigiu o manuscrito, delineou o método e aprovou a versão final. Mendes KMB redigiu o manuscrito, delineou o método e aprovou a versão final. Pereira AP fez a revisão de literatura e aprovou a versão final. Dias LC fez a revisão de literatura e aprovou a versão final. Menezes CS redigiu o manuscrito e aprovou a versão final.

\section{Conflitos de interesses}

Nenhum conflito financeiro, legal ou político envolvendo terceiros (governo, empresas e fundações privadas, etc.) foi declarado para nenhum aspecto do trabalho submetido (incluindo mas não limitando-se a subvenções e financiamentos, participação em conselho consultivo, desenho de estudo, preparação de manuscrito, análise estatística, etc.). 


\section{Referências}

1. Epstein SK, Ciubotaru RL, Wong JB. Effect of failed extubation on the outcome of mechanical ventilation. Chest. 1997;112(1):186-92. doi: $10.1378 /$ chest.112.1.186

2. Ferrer M, Sellarés J, Valencia M, Carrillo A, Gonzalez G, Badia $J R$ et al. Non-invasive ventilation after extubation in hypercapnic patients with chronic respiratory disorders: randomised controlled trial. The Lancet. 2009;374(9695):1082-8. doi: 10.1016/ S0140-6736(09)61038-2

3. Nava S, Gregoretti C, Fanfulla F, Squadrone E, Grassi M, Carlucci $A$ et al. Noninvasive ventilation to prevent respiratory failure after extubation in high-risk patients. Crit Care Med. 2005;33(11):24652470. doi: $10.1097 / 01 . c c m .0000186416 .44752 .72$

4. Ferrer M, Valencia M, Nicolas JM, Bernadich O, Badia JR, Torres A. Early noninvasive ventilation averts extubation failure in patients at risk: a randomized trial. Am J Respir Crit. Care Med. 2006;173(2):164-170. doi: 10.1164/rccm.200505-7180C

5. Antonelli M, Bello G. Noninvasive mechanical ventilation during the weaning process: facilitative, curative, or preventive?. Crit Care. 2008;12(2):136. doi: 10.1186/cc6853

6. Rochwerg B, Brochard L, Elliott MW, Hess D, Hill NS, Nava S et al. Official ERS/ATS clinical practice guidelines: noninvasive ventilation for acute respiratory failure. Eur Respir J. 2017;50(2):1602426. doi: 10.1183/13993003.02426-2016

7. Khilnani GC, Galle AD, Hadda V, Sharma SK. Non-invasive ventilation after extubation in patients with chronic obstructive airways disease: a randomised controlled trial. Anaesth Intensive Care. 2011;39(2): 217-223. doi: 10.1177/0310057X1103900210

8. Su CL, Chiang LL, Yang SH, Lin HY, Cheng KC, Huang YCT et al. Preventive use of noninvasive ventilation after extubation: a prospective, multicenter randomized controlled trial. Respir care. 2012;57(2):204-210. doi: 10.4187/respcare.01141

9. Mohamed KAE, Abdalla MH. Role of non invasive ventilation in limiting re-intubation after planned extubation. Egypt J Chest Dis Tuberc. 2013;62(4):669-674. doi: 10.1016/j.ejcdt.2013.08.010

10. Ornico SR, Lobo SM, Sanches HS, Deberaldini M, Tófoli LT, Vidal AM et al. Noninvasive ventilation immediately after extubation improves weaning outcome after acute respiratory failure: a randomized controlled trial. Crit. Care. 2013;17(2):R39. doi: $10.1186 /$ cc12549

11. Adıyeke E, Ozgultekin A, Turan G, Iskender A, Canpolat G, Pektaş A et al. Ventilação mecânica não invasiva após desmame bem-sucedido: uma comparação com a máscara de Venturi. Braz J Anesthesiol. 2016;66(6):572-576. doi: 10.1016/j.bjane.2017.08.005
12. Plant PK, Owen JL, Elliott MW. Non-invasive ventilation in acute exacerbations of chronic obstructive pulmonary disease: long term survival and predictors of in-hospital outcome. Thorax. 2001;56(9):708-712. doi: 10.1136/thorax.56.9.708

13. Jhonghe B, Bastuji-Garin S, Durand M, Malissin I, Rodrigues $P$, Cerf $C$ et al. Respiratory weakness is associated with limb weakness and delayed weaning in critical illness. Crit Care Med. 2007;35(9):2007-15. doi: 10.1097/01.ccm.0000281450.01881.d8

14. Yang KL, Tobin MJ. A prospective study of indexes predicting the outcome of trials of weaning from mechanical ventilation. N Engl J Med. 1991;324(21):1445-50. doi: 10.1056/ NEJM199105233242101 